\title{
Does Warm-Up Have a Beneficial Effect on 100-m Freestyle?
}

\author{
Henrique P. Neiva, Mario C. Marques, Ricardo J. Fernandes, João L. Viana, \\ Tiago M. Barbosa, and Daniel A. Marinho
}

\begin{abstract}
Purpose: To investigate the effect of warm-up on 100-m swimming performance. Methods: Twenty competitive swimmers (with a training frequency of $8.0 \pm 1.0$ sessions/wk) performed 2 maximal $100-\mathrm{m}$ freestyle trials on separate days, with and without prior warm-up, in a counterbalanced and randomized design. The warm-up distance totaled $1000 \mathrm{~m}$ and replicated the swimmers' usual precompetition warm-up strategy. Performance (time), physiological (capillary blood lactate concentrations), psychophysiological (perceived exertion), and biomechanical variables (distance per stroke, stroke frequency, and stroke index) were assessed on both trials. Results: Performance in the $100-\mathrm{m}$ was fastest in the warm-up condition $(67.15 \pm 5.60$ vs $68.10 \pm 5.14 \mathrm{~s} ; P$ $=.01)$, although 3 swimmers swam faster without warm-up. Critical to this was the $1 \mathrm{st} 50-\mathrm{m}$ lap $(32.10 \pm$ 2.59 vs $32.78 \pm 2.33 \mathrm{~s} ; P<.01)$, where the swimmers presented higher distance per stroke $(2.06 \pm 0.19$ vs. $1.98 \pm 0.16 \mathrm{~m} ; P=.04$ ) and swimming efficiency compared with the no-warm-up condition (stroke index $3.46 \pm 0.53$ vs $3.14 \pm 0.44 \mathrm{~m}^{2} \cdot \mathrm{c}^{-1} \cdot \mathrm{s}^{-1} ; P<.01$ ). Notwithstanding this better stroke-kinematic pattern, blood lactate concentrations and perceived exertion were similar between trials. Conclusions: These results suggest that swimmers' usual warm-up routines lead to faster 100-m freestyle swimming performance, a factor that appears to be related to better swimming efficiency in the 1st lap of the race. This study highlights the importance of performing swimming drills (for higher distance per stroke) before a maximal 100-m freestyle effort in similar groups of swimmers.
\end{abstract}

Keywords: preexercise, performance, kinetics, efficiency, lactate

Although there is a lack of conclusive scientific evidence, the use of warm-up to enhance performance seems to be a matter of common belief and practice among coaches and athletes. In fact, the different physical exercises performed during warm-up aim to increase muscle and core temperature and, through the body's underlying mechanisms, improve performance. ${ }^{1-4}$ The hyperthermia resulting from physical activity increases vasodilatation and muscle blood flow, stimulating the increased aerobic energy contribution during a subsequent task. ${ }^{3,5}$ In addition, it increases the muscle glycogenolysis, glycolysis, and high-energy phosphate degradation during exercise. ${ }^{6}$ The literature also claims that warming up via physical activity might have effects additional to the increase in temperature, particularly an elevation of baseline of oxygen consumption $\left(\mathrm{VO}_{2}\right)$ and of the amplitude of the primary $\mathrm{VO}_{2}$ response in the subsequent exercise. ${ }^{7}$ Nevertheless, although these metabolic responses appear to indicate a positive effect

Neiva, Marques, Viana, and Marinho are with the Dept of Sport Sciences, University of Beira Interior, Covilhã, Portugal. Fernandes is with the Faculty of Sport, University of Porto, Porto, Portugal. Barbosa is with the National Inst of Education, Nanyang Technological University, Singapore. of warm-up on athletic performance, current evidence is still inconclusive. ${ }^{1,2}$

Specifically in swimming, different physiological changes and conflicting benefits to performance have been reported. Houmard et $\mathrm{al}^{8}$ described increments in distance per stroke (DPS) during an intensely paced 368.5-m swim and decreased postexercise blood lactate concentrations $\left(\left[\mathrm{La}^{-}\right]\right)$with warm-up. Conversely, others ${ }^{9}$ found that warm-up procedures did not change performance and led to higher $\left[\mathrm{La}^{-}\right]$after a 2-minute high-intensity swimming trial $(13.66 \pm 2.66$ vs $9.53 \pm$ $2.22 \mathrm{mmol} / \mathrm{L}$ ). Regarding a shorter-distance swimming performance, studies have shown that proper warm-up was effective in reducing 100 -yd time trial by $0.44^{10}$ and 0.75 seconds ${ }^{11}$ compared with a bout without prior warm-up, but Bobo ${ }^{12}$ failed to find significant differences in 100-yd performance between 3 conditions (warm-up exercises in water and on dry land and without warm-up).

More recent research has focused on even shorter distances (50 yd and $50 \mathrm{~m}$ ), but results are inconclusive; no favorable effects of warm-up on 50-m front-crawl performance, either in the $\left[\mathrm{La}^{-}\right]$or perceived exertion (RPE), were observed by Neiva et al, ${ }^{13}$ but Balilionis et $\mathrm{al}^{14}$ reported better performances on 50 -yd freestyle after a warm-up ( 0.2 s), although no effects on RPE and stroke frequency $(\mathrm{SF})$ were detected. 
Given the lack of consistent evidence about the effects of warm-up on swimming performance, the purpose of the current study was to investigate if usual warm-up procedures are beneficial to 100-m freestyle swimming performance. Performance (time), biomechanical (SF, DPS, and stroke index [SI]), physiological ([La $]$ ), and psychophysiological (RPE) variables were assessed. Usual warm-up was hypothesized to positively contribute to the swimmers' response to maximal 100-m freestyle performance.

\section{Methods}

\section{Subjects}

Twenty competitive swimmers (10 male and 10 female: age $16.0 \pm 0.6$ and $16.2 \pm 1.14 \mathrm{y}$, height $173 \pm 5.07$ and $161 \pm 7.04 \mathrm{~cm}$, body mass $62.3 \pm 3.9$ and $55.9 \pm 6.3 \mathrm{~kg}$, respectively) volunteered to participate in this study. The swimmers had been engaged in competitive swimming during the last $7.11 \pm 1.29$ years and had a training frequency of $8.0 \pm 1.0(16.0 \pm 1.5 \mathrm{~h})$ sessions per week, with a training volume of $34,000 \pm 5400 \mathrm{~m} / \mathrm{wk}$ during the current season. Personal-best time in the 100-m freestyle event was $64.71 \pm 5.43$ seconds, which corresponds to $456.70 \pm 85.91$ FINA 2011 points scoring. Before the experiments, swimmers were informed about the study design and procedures, and an informed consent was signed. Institutional review board approval was granted, in the spirit of the Helsinki declaration.

\section{Experimental Design}

All experiments were conducted 1 week after the main competition of the season in a 50-m indoor swimming pool with water temperature at $27.5^{\circ} \mathrm{C}$. The swimmers performed two 100-m freestyle time trials at maximum velocity with (WU) and without (NWU) prior warm-up, with a counterbalanced order of treatments and random assignment to order. Trials were separated by 24 hours. Each swimmer performed the 100 -m as an individual time trial to prevent pacing or tactic effects. Swimmers were asked to wear the swimsuits they normally wore during competitions.

In the WU trial, swimmers performed their usual precompetition warm-up (Table 1), comprising $1000 \mathrm{~m}$ of aquatic drills, pull and kick exercises, and specific sets. After 10 minutes of passive rest, they performed the 100-m freestyle time trial. In the NWU trial, no physical activity was allowed previous to the 100-m freestyle time trial.

\section{Methodology}

In both the WU and NWU conditions, in-water starts were used, and 100-m times for each swimmer were registered by 2 experienced coaches using stopwatches (Golfinho Sports MC 815, Aveiro, Portugal). The mean value of the 2 times was recorded for analysis. Coaches were blind as to the warm-up conditions of the swimmers.
Table 1 Usual Warm-Up Protocol

\begin{tabular}{ll}
\hline Distance & Intensity/Exercise \\
\hline $300 \mathrm{~m}$ & Easy swim \\
$2 \times 100 \mathrm{~m} / 15$-s rest & $\begin{array}{l}\text { (Second faster, higher distance per } \\
\text { stroke) }\end{array}$ \\
$8 \times 50 @ 1 \mathrm{~min}$ & 25 -m kick/25-m complete, $2 \times$ \\
& 25 -m drills/25-m complete, $2 \times$ \\
& 25 -m race pace/25 easy, $2 \times$ \\
& 25 -m race pace/25 easy, $2 \times$ \\
& Easy swim \\
\hline
\end{tabular}

Swimming velocity was determined in the middle $15 \mathrm{~m}$ of the swimming pool (marks were set at 20 and $35 \mathrm{~m}$ ). The distance covered by a swimmer was divided by the time spent to cover such distance. SF was measured with a chronofrequency meter (Golfinho Sports MC 815, Aveiro, Portugal) from 3 consecutive stroke cycles within the same $15 \mathrm{~m}$ where swimming velocity was assessed. Afterward, SF was converted to International System units $(\mathrm{Hz})$. The velocity and SF were assessed by 2 different and experienced researchers, who were also blind to the swimmers' testing condition. Intraclass correlation coefficient was determined for the time, velocity, and SF, to ensure the accuracy of the measurement. The mean value was used for analysis. DPS was estimated as the division between the velocity obtained during the 15-m and the SF. ${ }^{15}$ The SI was computed as the product of the velocity of the swimmer during the $15-\mathrm{m}$ recorded and the corresponding DPS. ${ }^{16}$

Capillary blood samples for [ $\left.\mathrm{La}^{-}\right]$assessment (Accutrend LactateRoche, Germany) were collected from the fingertip after each maximal trial (at the first and third minutes of recovery) to determine its higher value. RPEs were registered after each test using Borg's 6- to 20 -point scale. ${ }^{17}$

\section{Statistical Analysis}

Standard statistical methods were used for calculation of mean and SD for all variables. The normality of all distributions was verified using Shapiro-Wilk tests, and parametric statistical analysis was adopted. To compare data obtained in the 2 trials, Student paired $t$ tests were used. Limits of agreement between the performance measured in WU and NWU were derived according to the literature. ${ }^{18}$ Statistical procedures were performed using SPSS 19.0 for Windows (Chicago, IL, USA). Post hoc analysis of power $(1-\beta)$ and the values of Cohen $d$ effect size for repeated measures (ES) were accomplished using G-Power 3.1.3 for Windows (University of Kiel, Germany). The level of statistical significance was set at $P \leq .05$. 


\section{Results}

Table 2 presents a comparison between the 100-m freestyle performance, the respective lap times, and the values of [La $]$, RPE, SF, DPS, and SI recorded in the first and second $50 \mathrm{~m}$ in the WU and NWU conditions. In the $100-\mathrm{m}$ WU trial, the swimmers were $1.48 \% \pm 2.06 \%$ faster, with medium magnitudes of differences $(\mathrm{ES}>0.5)$ resulting from the large ES $(>0.8)$ noted in the first 50-m lap.

It is also possible to observe $\left[\mathrm{La}^{-}\right]$higher than 10 $\mathrm{mmol} / \mathrm{L}$ and hard to very hard effort $(\mathrm{RPE}>16)$ in both testing conditions. The differences verified in the second $50-\mathrm{m} \mathrm{SF}$, in the first 50-m and second 50-m DPS, and in the first 50-m SI between the WU and NWU conditions ranged from a medium $(.5<d<.8)$ to a large $(d>.8)$ magnitude of the effect.

The individual differences between WU and NWU on the first and second 50-m laps and for the 100-m total performance are presented in Figure 1, evidencing some individual positive responses after NWU in figure parts $\mathrm{A}, \mathrm{B}$, and $\mathrm{C}(10 \%, 25 \%$, and $15 \%$, respectively), although the mean value is positioned below zero.

Figure 2 presents the variation of the biomechanical variables between the 50-m laps of the 100-m maximal effort for WU and NWU. It should be noted that the lower the value presented, the more inferior were the values obtained in the second 50-m lap. Differences were observed in the patterns of SF, DPS, and SI when maximal 100-m freestyle was preceded by WU or NWU.

\section{Discussion}

The purpose of the current study was to investigate the effects of warm-up on maximal 100-m freestyle swimming performance. The faster times observed in the
WU trial suggest that the swimmers' usual warm-up procedures have a beneficial effect on their subsequent swimming performance. Warming up led to significant improvements in the stroke mechanics, yet the assessed physiological and psychophysiological variables did not seem to be influenced. These findings evidence the positive influence that usual warm-up may have on swimming performance, which appears to be mostly related to the swimmers' technical pattern.

Warming up before the competition is a usual practice in swimming. Its positive effect was first presented by De Vries, ${ }^{10}$ but recent literature focusing specifically on short swimming distances demonstrated that priming exercises could impair swimming performance..$^{9,12,13}$ It should be noted that in most swimming competitions, there is a considerable time lapse between the in-water warm-up and the competitive event, which can negate the warm-up's possible beneficial effects. Nevertheless, swimmers still compete at their maximum effort. These findings and considerations highlight the relevance of better understanding the warm-up phenomenon.

The lack of previous research assessing the effects of warm-up on maximal 100-m swimming limits comparisons with the current study results. In-water start and the lack of competitive context during the tests might explain the slightly higher final times compared with swimmers' personal bests ( 2.43 s) and with the literature. ${ }^{19,20}$ As the 2 experimental tests were performed in the same competitive environment, these effects could be disregarded. Furthermore, the RPE values obtained were similar to those in previous studies that assessed all-out swimming performances, ${ }^{13,14,21}$ which could ensure the reliability of the results obtained.

Concerning the main aim of the current study, faster 100-m performances were achieved in the WU condition,

Table 2 Results for Tested Parameters in the 100-m Trial, $\mathbf{N}=\mathbf{2 0}$

\begin{tabular}{|c|c|c|c|c|c|}
\hline & \multicolumn{2}{|c|}{ Condition, Mean \pm SD } & \multirow[b]{2}{*}{$\boldsymbol{P}$} & \multirow[b]{2}{*}{ ES } & \multirow[b]{2}{*}{$1-\beta$} \\
\hline & Warm-up & No warm-up & & & \\
\hline $100-\mathrm{m}$ time $(\mathrm{s})^{* *}$ & $67.15 \pm 5.60$ & $68.10 \pm 5.14$ & .01 & 0.69 & .99 \\
\hline First 50 -m time $(\mathrm{s})^{* *}$ & $32.10 \pm 2.59$ & $32.78 \pm 2.33$ & $<.01$ & 0.89 & 1.00 \\
\hline Second 50-m time (s) & $35.00 \pm 3.27$ & $35.37 \pm 2.98$ & .07 & 0.44 & .76 \\
\hline Blood lactate concentration (mmol/L) & $10.91 \pm 1.75$ & $10.28 \pm 2.20$ & .22 & 0.32 & .41 \\
\hline $100-\mathrm{m}$ rating of perceived exertion & $16.90 \pm 1.80$ & $16.10 \pm 1.55$ & .08 & 0.41 & .71 \\
\hline Stroke frequency, first $50 \mathrm{~m}(\mathrm{~Hz})$ & $0.81 \pm 0.07$ & $0.80 \pm 0.06$ & .25 & 0.28 & .37 \\
\hline Stroke frequency, second $50 \mathrm{~m}(\mathrm{~Hz})^{* *}$ & $0.77 \pm 0.60$ & $0.72 \pm 0.06$ & $<.01$ & 1.09 & 1.00 \\
\hline Distance per stroke, first $50 \mathrm{~m}(\mathrm{~m})^{*}$ & $2.06 \pm 0.19$ & $1.98 \pm 0.16$ & .04 & 0.53 & .88 \\
\hline Distance per stroke, second $50 \mathrm{~m}(\mathrm{~m})^{* *}$ & $1.90 \pm 0.18$ & $1.99 \pm 0.18$ & .01 & 0.66 & .97 \\
\hline Stroke index, first $50 \mathrm{~m}\left(\mathrm{~m}^{2} \cdot \mathrm{c}^{-1} \cdot \mathrm{s}^{-1}\right)^{* *}$ & $3.46 \pm 0.53$ & $3.14 \pm 0.44$ & $<.01$ & 0.87 & 1.00 \\
\hline Stroke index, second $50 \mathrm{~m}\left(\mathrm{~m}^{2} \cdot \mathrm{c}^{-1} \cdot \mathrm{s}^{-1}\right)$ & $2.81 \pm 0.46$ & $2.89 \pm 0.45$ & .22 & 0.29 & .42 \\
\hline
\end{tabular}

$* P \leq .05 . * * P \leq .01$. 

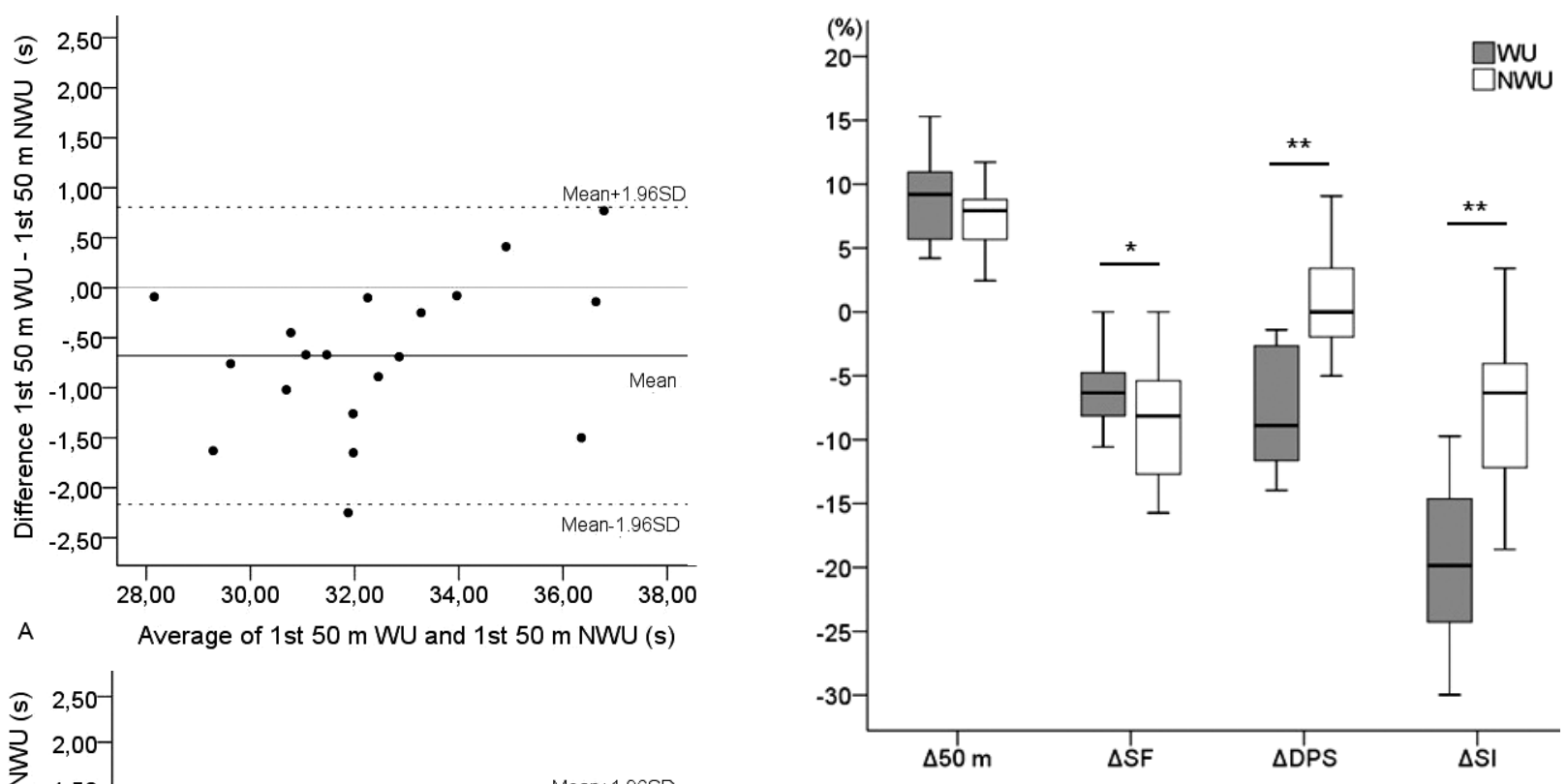

Figure 2 - Comparison between the variations of the time $(\Delta 50 \mathrm{~m})$, stroke frequency $(\Delta \mathrm{SF})$, distance per stroke $(\Delta \mathrm{DPS})$, and stroke index $(\Delta \mathrm{SI})$ assessed in the first and second $50-\mathrm{m}$ laps of the $100-\mathrm{m}(\Delta=$ second - first $)$, with warm-up (WU) and without warm-up (NWU). ${ }^{*} P \leq .05, * * P \leq .01, \mathrm{~N}=20$

those differences being mainly achieved in the first lap. According to Balilionis et $\mathrm{al},{ }^{14}$ it is expected that swimmers will be faster in the 50 -yd freestyle when using a regular warm-up ( 1300 yd) than when using a shorter warm-up (100 yd) or when no warm-up is used (24.95 \pm $1.53,25.26 \pm 1.61$, and $25.19 \pm 1.54 \mathrm{~s})$. The differences between the 2 conditions disappeared in the second $50-\mathrm{m}$ lap, even though the observed medium effect size requires further investigation under this topic.

The results presented in Table 2 are confirmed in Figure 1 since the average difference line is farther away from zero in figure parts $\mathrm{A}$ and $\mathrm{C}$ than in part $\mathrm{B}$. However, the figure also illustrates individual cases in which the difference was positive, evidencing that these swimmers reacted favorably to the nonexistence of prior exercises ( 2 in the first $50 \mathrm{~m}, 5$ in the second $50 \mathrm{~m}$, and 3 in the total $100 \mathrm{~m}$ ). This illustrates the individuality of swimming, as there is no clear zone in which the swimmers can be placed. Therefore swimmer individuality should be respected, and coaches should take that into account when defining their training process and determining competitive procedures.

During a $100-\mathrm{m}$ swimming event the anaerobic metabolism is a substantial source of energy, leading to $\left[\mathrm{La}^{-}\right]$higher than $10 \mathrm{mmol} / \mathrm{L},{ }^{22}$ as observed in our data. Warm-up has been proposed to maintain the acid-base balance at an appropriate level by stimulating the buffering capacity, ${ }^{23}$ which was evidenced after maximal $200-\mathrm{m}$ swimming: net $\left[\mathrm{La}^{-}\right]$of $8.7 \pm 0.9$ versus $10.9 \pm 0.5 \mathrm{mmol} / \mathrm{L}$ $(P<.05)$, with and without warm-up, respectively. ${ }^{24}$ However, in the current study, $\left[\mathrm{La}^{-}\right]$levels were similar

Figure 1 - Bland-Altman plots representing (A) the first 50-m-lap time, (B) the second 50-m-lap time, and (C) 100-m total time in the 2 trial conditions, with warm-up (WU) and without warm-up (NWU). Average difference (solid line) and $95 \% \mathrm{CI}$ (dashed lines) are indicated $(\mathrm{N}=20)$. 
in the WU and NWU conditions, which is consistent with recent findings for short-distance swimming. ${ }^{13,25}$ Possibly, different physiological variables $\left(\mathrm{eg}, \mathrm{VO}_{2}\right.$ ) are influenced to a greater extent by warm-up procedures. As $100-\mathrm{m}$ swimming performance also relies on the aerobic energy system, ${ }^{26}$ the warm-up could allow enhancement of the swimmers' aerobic system more than their anaerobic one.

Swimmers in general believe that warm-up is essential to attain a good performance, ${ }^{27}$ so psychological beliefs could influence the perception of effort exerted. However, the observed RPE values were similar in WU and NWU conditions, as reported before. . $^{813,14,25}$ The RPE is assumed to be influenced by fatigue perception mainly due to the accumulation of hydrogen ions in the active muscles as the result of the dissociation of lactic acid. ${ }^{28}$ Hence, given that $\left[\mathrm{La}^{-}\right]$values did not change between WU and NWU, it was somewhat expected that RPE would also be similar between conditions.

The faster initial meters of the 100-m freestyle in the WU condition reflected the higher DPS and, consequently, greater swimmer efficiency (evidenced by SI). Therefore, in the NWU condition it is expected that the swimmers would not be effective in the arm pull and were technically compromised, ${ }^{20}$ lowering their DPS in the first 50-m lap. However, in the second 50-m lap, and once the velocity is related to SF and DPS, ${ }^{29}$ the higher DPS and lower SF with NWU dissipated the differences in the time performed between conditions. In NWU, the swimmers were able to maintain the DPS in the second $50 \mathrm{~m}$, maybe as a consequence of the lower energy cost and velocity of the first meters, but the SF experienced a further reduction as the fatigue increased.

The variations of SF, DPS, and SI throughout the 100-m freestyle in the WU and NWU conditions could reflect the development of fatigue. According to Barbosa et $\mathrm{al},{ }^{29}$ swimmers are able to manipulate their DPS and SF to achieve a given velocity with the lowest energy cost. Thus, the swimmers used different kinematic patterns in the 2 experimental conditions to accomplish the total distance at maximum intensity according to their energetic needs.

\section{Practical Applications}

The usual warm-up performed by the swimmers was effective in optimizing 100-m freestyle swimming performance. Perhaps the greater swimming efficiency verified in the first meters suggests that incorporating technical drills during warm-up could be beneficial to similar groups of swimmers, specifically regarding their DPS. Some positive responses to NWU revealed the swimmers' individuality, and this confirms the idea that warm-up procedures should be considered as an individualized approach to optimizing swimmer performance. ${ }^{14}$ Thus, there is no single model that should be copied and adopted by all swimmers. It is fundamental to consider their biological individualities, and group procedures should be handled with caution at the risk of compromising optimal performances. Possibly, swimmers and coaches need to test over several occasions to establish consistent responses to different warm-up procedures and thereby establish their own optimal warm-up. The unknown value of the variation in performance day to day or test to test limited the understanding of the magnitude of the effects of warm-up. However, the results are clear in demonstrating a positive effect, and future research is needed to better understand the ideal structure of warm-up procedures.

\section{Conclusion}

The swimmers were significantly faster in the first $50-\mathrm{m}$ lap of the WU trial, which led to an improvement in overall 100-m performance. Different biomechanical patterns were observed in response to the WU and NWU conditions, suggesting that warm-up significantly influences the stroke patterns of a short swimming event. The individuality of the swimmers was also shown, enhancing the importance of an individualized approach to optimizing swimming performance.

\section{Acknowledgments}

The authors would like to acknowledge the help of the swimmers who participated in the current study, and also that of the Varzim Lazer EEM in making available the swimming-pool facilities. The Portuguese government supported this work by way of a grant of the Science and Technology Foundation (SFRH/BD/74950/2010). This work was also supported by University of Beira Interior and Santander Totta Bank (UBI/ FCSH/Santander/2010).

\section{References}

1. Bishop D. Warm up I: potential mechanisms and the effects of passive warm up on exercise performance. Sports Med. 2003a;33(6):439-454. PubMed doi:10.2165/00007256200333060-00005

2. Bishop D. Warm up II: performance changes following active warm up and how to structure the warm up. Sports Med. 2003b;33(7):483-498. PubMed doi:10.2165/00007256-200333070-00002

3. Gray S, Nimmo M. Effects of active, passive or no warmup on metabolism and performance during high-intensity exercise. J Sports Sci. 2001;19(9):693-700. PubMed doi:10.1080/02640410152475829

4. Wright V, Johns RJ. Quantitative and qualitative analysis of joint stiffness in normal subjects and in patients with connective tissue diseases. Ann Rheum Dis. 1961;20(1):36-46. PubMed doi:10.1136/ard.20.1.36

5. Pearson J, Low DA, Stöhr E, et al. Hemodynamic responses to heat stress in the resting and exercising human leg: insight into the effect of temperature on skeletal muscle blood flow. Am J Physiol Regul Integr Comp Physiol. 2011;300(3):R663-R673. PubMed doi:10.1152/ ajpregu.00662.2010

6. Febbraio MA, Carey MF, Snow RJ, Stathis CG, Hargreaves $\mathrm{M}$. Influence of elevated muscle temperature on metabolism during intense, dynamic exercise. Am J Physiol. 1996;271(5 Pt 2):R1251-R1255. PubMed 
7. Burnley M, Davison G, Baker JR. Effects of priming exercise on $\mathrm{VO}_{2}$ kinetics and the power-duration relationship. Med Sci Sports Exerc. 2011;43(11):2171-2179. PubMed doi:10.1249/MSS.0b013e31821ff26d

8. Houmard JA, Johns RA, Smith LL, Wells JM, Kobe RW, McGoogan SA. The effect of warm-up on responses to intense exercise. Int J Sports Med. 1991;12(5):480-483. PubMed doi:10.1055/s-2007-1024717

9. Mitchell JB, Huston JS. The effect of high- and lowintensity warm-up on the physiological responses to a standardised swim and tethered swimming performance. J Sports Sci. 1993;11(2):159-165. PubMed doi:10.1080/02640419308729979

10. De Vries HA. Effects of various warm-up procedures on 100-yard times of competitive swimmers. Res $Q$. 1959;30:11-22.

11. Romney NC, Nethery VM. The effects of swimming and dryland warm-ups on 100-yard freestyle performance in collegiate swimmers. J Swim Res. 1993;9:5-9.

12. Bobo M. The effect of selected types of warm-up on swimming performance. Int Sports J. 1999;3(2):37-43.

13. Neiva HP, Morouço PG, Pereira FM, Marinho DA. The effect of warm-up in $50 \mathrm{~m}$ swimming performance. Rev Mot. 2012;8(S1):13-18.

14. Balilionis G, Nepocatych S, Ellis CM, Richardson MT, Neggers YH, Bishop PA. Effects of different types of warm-up on swimming performance, reaction time, and dive distance. J Strength Cond Res. 2012;26(12):32973303. PubMed doi:10.1519/JSC.0b013e318248ad40

15. Craig AB, Pendergast DR. Relationships of stroke rate, distance per stroke, and velocity in competitive swimming. Med Sci Sports. 1979;11(3):278-283. PubMed

16. Costill DL, Kovaleski J, Porter D, Fielding R, King D. Energy expenditure during front crawl swimming: predicting success in middle-distance events. Int J Sports Med. 1985;6:266-270. PubMed doi:10.1055/s-2008-1025849

17. Borg G, ed. Borg's Perceived Exertion and Pain Scales. Champaign, IL: Human Kinetics; 1998.

18. Bland JM, Altman DG. Applying the right statistics: analyses of measurement studies. Ultrasound Obstet Gynecol. 2003;22(1):85-93. PubMed doi:10.1002/uog.122
19. Stirn I, Jarm T, Kapus V, Strojnik V. Evaluation of muscle fatigue during 100-m front crawl. Eur J Appl Physiol. 2011;111(1):101-113. PubMed doi:10.1007/s00421-0101624-2

20. Toussaint HM, Beek PJ. Biomechanics of competitive front crawl swimming. Sports Med. 1992;13(1):8-24. PubMed doi:10.2165/00007256-199213010-00002

21. Zochowski T, Johnson E, Sleivert GG. Effects of varying post-warm-up recovery time on 200-m time-trial swim performance. Int J Sports Physiol Perform. 2007;2(2):201211. PubMed

22. Bonifazi M, Marteli G, Marugo L, Sardella F, Cari G. Blood lactate accumulation in top level swimmers following competition. J Sports Med Phys Fitness. 1993;33(1):13-18. PubMed

23. Beedle BB, Mann CL. A comparison of two warmups on joint range of motion. J Strength Cond Res. 2007;21(3):776-779. PubMed

24. Robergs RA, Costill DL, Fink WJ, et al. Effects of warmup on blood gases, lactate and acid-base status during sprint swimming. Int J Sports Med. 1990;11(4):273-278. PubMed doi:10.1055/s-2007-1024806

25. Neiva H, Morouço P, Silva AJ, Marques MC, Marinho DA. The effect of warm up on tethered front crawl swimming forces. J Hum Kinet. 2011;(Spec Iss):113-119.

26. Capelli C, Pendergast D, Termin B. Energetics of swimming at maximal speeds in humans. Eur J Appl Physiol Occup Physiol. 1998;78:385-393. PubMed doi:10.1007/s004210050435

27. De Bruyn-Prevost P. The effects of various warming up intensities and durations upon some physiological variables during an exercise corresponding to the WC170. Eur J Appl Physiol Occup Physiol. 1980;43(2):93-100. PubMed doi:10.1007/BF00422439

28. Robertson RJ, Falkel JE, Drash AL, et al. Effect of blood $\mathrm{pH}$ on peripheral and central signals of perceived exertion. Med Sci Sports Exerc. 1986;18(1):114-122. PubMed doi:10.1249/00005768-198602000-00019

29. Barbosa TM, Fernandes RJ, Keskinen KL, Vilas-Boas JP. The influence of stroke mechanics into energy cost of elite swimmers. Eur J Appl Physiol. 2008;103(2):139-149. PubMed doi:10.1007/s00421-008-0676-Z 